Methods The frequencies of Th22, Th17, Th1 cells were determined by flow cytometry of peripheral blood by the chemokine receptors or/and the intracellular cytokine from a total of 25 patients with freshly diagnosed SLE and 13 age-/gendermatched healthy controls, and the values were compared with disease activity as determined by the Systemic Lupus Erythematosus Disease Activity (SLEDAI), serum complement factors (C3, C4), C-reactive protein (CRP), Erythrocyte sedimentation rate(ESR), Immunoglobulin(Ig), anti-double stranded (ds) DNA and anti-Smith $(\mathrm{Sm})$ antibodies were measured.

Results We found increased Th22, Th17 cells in SLE patients compared with those in healthy controls. The elevated Th22 positive correlated with SLEDAI, ESR, IgG and IgA. Higher frequencies of Th22 and positive correlations between the percentage of Th22 cells and Revised Cutaneous Lupus Erythematosus Disease Area and Severity Index (RCLASI) were observed in patients with lupus skin disease.

Conclusions Our data suggests that both Th22 and Th17 may participate in the pathogenesis of SLE and Th22 may migrate to skin and promote inflammation in the lupus skin impairment.

\section{ALTERED PROPORTION OF TFH17 SUBSETS IN BRONCHOLALVEOLAR LAVAGE FLUID OF PATIENTS WITH INTERSTITIAL LUNG DISEASE CAUSED BY SYSTEMIC LUPUS ERYTHEMATOSUS}

${ }^{1} \mathrm{Q}$ Zhou*, ${ }^{1} \mathrm{~J} \mathrm{Su},{ }^{2} \mathrm{~J}$ Tian, ${ }^{3} \mathrm{G}$ Tang, ${ }^{1} \mathrm{~B}$ Zhou. ${ }^{1}$ Sichuan Academy of Medical Sciences Sichuan Provincial People's Hospital, Rheumatology and Immunology, Chengdu, China; ${ }^{2}$ North Sichuan Medical College, Institute of Rheumatology, Nanchong, China; ${ }^{3} Z$ unyi Medical University, Institute of Rheumatology, Zunyi, China

\subsection{6/lupus-2017-000215.58}

Background and aims Interstitial lung disease (ILD) is common in systemic lupus erythematosus (SLE) patients. These patients tend to have large amounts of autoantibodies. Blood memory Tfh17 cells represent efficient B cell helper cells with distinct capacities to induce $\operatorname{IgG}$ and $\operatorname{IgA}$ secretion and to regulate immunoglobulin isotope switching. Recent study found overall Tfh17 cells are highly presented in peripheral blood of SLE patients. However, it is not clear how different subsets of Tfh17 cell are distributed in broncholalveolar lavage fluid (BAL) and peripheral blood of SLE-ILD patients. The study is to determine the proportion of different Tfh17 cell subsets (activated Tfh17: CXCR3-CCR6+ICOS+PD-1++CCR7lo, quiescent: CXCR3-CCR6+ICOS-PD-1+CCR7int and CXCR3CCR6+ICOS-PD-1-CCR7hi) among CD4+ T cells and levels of immunoglobulins in BAL and peripheral blood of SLE-ILD patients.

Methods 30 SLE-ILD patients were included. The lung disease were proved by high resolution CT scan. Patients underwent bronchoscopy and BAL were collected. Tfh17 cell profiles were determined using flow cytometry. Levels of immunoglobulins were detected by ELISA. Statistics were analysed by SPSS 22.0.

Results IgA and IgG levels were significantly higher in BAL than in blood. Activated Tfh17 in BAL was increased significantly $(p=0.011)$ and both subsets of quiescent Tfh17 cells were decreased $(p<0.05)$ compared to those in the blood. The activated Tfh17 was positively correlated with IgA level $(r=0.871, p=0.039)$ in BAL and with $\operatorname{IgG}$ level $(r=0.714$, $\mathrm{p}=0.047)$ in blood.
Conclusions Activated Tfh 17 is more abundant in BAL than in blood and switches from IgG correlation to IgA correlation, suggesting its role in the pathogenesis of SLE-ILD.

\section{Antiphospholipid syndrome}

\section{ANTIPHOSPHOLIPID SYNDROME: ABOUT 62 CASES}

Z Aydi ${ }^{*}$ Z Hadj Ali, I Rachdi, F Daoud, H Zoubeidi, B BenDhaou, F Boussema. hôpital Habib Thameur, internal medcine, Tunis, Tunisia

\subsection{6/lupus-2017-000215.59}

Background and aims The antiphospholipid syndrome (SAPL) is an autoimmune and thrombogenic pathology that the diagnosis is based on clinical and biological criteria. It can be isolated (primary SAPL) or associated with another auto-immune disease (secondary SAPL). The purpose of this work is to finalise the epidemiological, clinical, biological, characteristic.

Methods We led a retrospective and descriptive study of the patients followed in the service of internal medicine for SAPL between January 1990 and April 2014.

Results We brought together 62 cases ( 61 women and 1 man). The average age was 41 years. The peripheral thromboses were observed in 51.6\%. The obstetric accidents were found at 26 patients dominated by repeated abortion $(35.5 \%)$ and fetal death in uterus (16.1\%). The cardiac infringement was dominated by valvular disease in $9.6 \%$. The lung demonstrations were represented by a pulmonary embolism in $32.25 \%$ and a lung arterial high blood pressure in $19.3 \%$. The neurological infringement was present in 29\%. The SAPL was primary in $32 \%$ and secondary in $86 \%$. The CAPS was found in 2 cases. The SLE was present in 59.7\%. The immunological balance sheet revealed aCL in $77.4 \%$, anti- $\beta 2$ GPI in $24.2 \%$ and anti-PT in $17.7 \%$. a statistically significant correlation between the obstetric and vascular sign with the presence of aCL.

Conclusions The SAPL is an entity among which the knowledge and the understanding are in permanent evolution. It is necessary to think of it in front of any vascular reccurent thrombosis to a young subject.

\section{SUBCLINICAL MYOCARDIAL DYSFUNCTION BY TISSUE DOPPLER ECHOCARDIOGRAPHY IN PRIMARY ANTIPHOSPHOLIPID SYNDROME: PRELIMINARY RESULTS}

'J Carvalho*, ${ }^{2}$ A Lianza. 'Brazil; ${ }^{2}$ Hospital das Clinicas da Faculdade de Medicina da USP, Echocardiography, Sao Paulo, Brazil

\subsection{6/lupus-2017-000215.60}

Background and aims To evaluate cardiac function in primary antiphospholipid syndrome (PAPS) patients using the echocardiogram with conventional and tissue Doppler evaluations.

Methods Nine PAPS patients (Sapporo criteria) were enrolled. Demographic and clinical data, co-morbidities, medication use and antiphospholipid antibodies were evaluated. All were asymptomatic regarding cardiovascular system. Exclusion criteria were history of heart failure, coronary artery disease, arrhythmia, valve abnormalities, age $>70$ years old, renal failure and severe hypertension. Seven age-, sex- and racematched healthy subjects were included as control group. 
Myocardial function was determined by echocardiogram (2-D, M-mode, tissue and conventional Doppler techniques).

Results Traditional cardiovascular risk factors were similar in PAPS and controls. PAPS patients had $55.6 \%$ of venous events, $55.6 \%$ arterial and $22.2 \%$ obstetric features, stroke was observed in $33.3 \%$, deep venous thrombosis in $44.4 \%$, livedo reticularis in $66.7 \%$. $88.9 \%$ were positive for IgG and/ or IgM anticardiolipina antibodies and $66.7 \%$ were positive for lupus anticoagulant. Conventional echocardiographic data was not altered in all parameters evaluated, comparing patients and controls. Regarding tissue Doppler echocardiogram data, a lower S' of lateral wall of left ventricle was observed in PAPS in comparison to controls [0.085 (0.007$0.12)$ vs. $0.12(0.09-0.13), p=0.004]$ as well as $A^{\prime}$ wave of the septum [0.07 $(0.06-0.08)$ vs. $0.09(0.07-0.11), p=0.02]$.

Conclusions Our data support the notion that PAPS patients have asymptomatic myocardial dysfunction evidenced by tissue Doppler echocardiography.

\section{ANTIPHOSPHOLIPID ANTIBODY SYNDROME PRESENTING AS SPONTANEOUS HEPATIC RUPTURE}

I Cunanan*, A Corpuz, VP Sta Maria, PA Casem. Ilocos Training and Regional Medical Centre, Internal Medicine, City of San Fernando, Philippines

10.1136/lupus-2017-000215.61

Background and aims Spontaneous hepatic rupture (SHR) is a rare peripartum complication and usually occurs among patients with pre-eclampsia, eclampsia or HELLP syndrome. We report a case of a 29 year old primigravid woman with fetal death in utero and spontaneous hepatic rupture secondary to undiagnosed Antiphospholipid Antibody Syndrome (APAS). Despite its uncommonness, a high index of suspicion

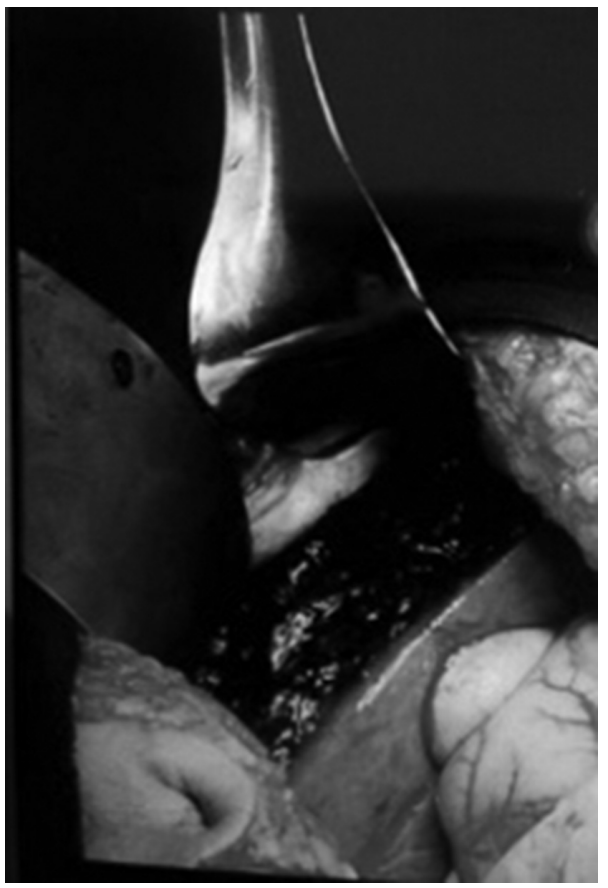

Abstract 61 Figure 1

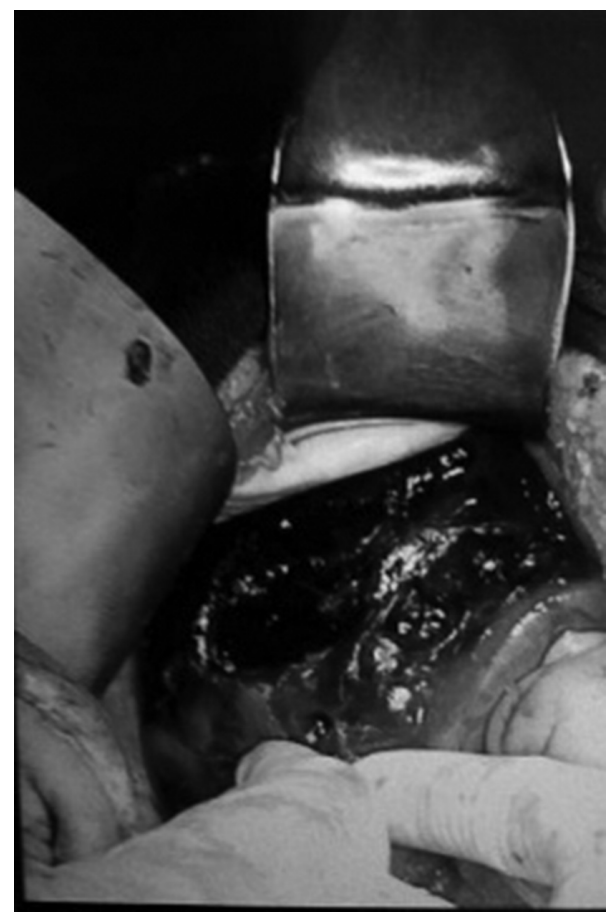

Abstract 61 Figure 2

Abstract 61 Table 1 Revised SAPPORO Classification criteris for the antiphospholipid antibody syndrome.

\begin{tabular}{|c|c|}
\hline & Clinical criteria \\
\hline \multirow[t]{2}{*}{$\checkmark$} & $\begin{array}{l}\text { 1. Vascular thrombosis } \\
\text { One or more clinical episodes of arterial, venous, or small vessel } \\
\text { thrombosis }\end{array}$ \\
\hline & 2. Pregnancy moromdity \\
\hline 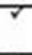 & $\begin{array}{l}\text { a. One of more unexplained feral deaths of a morphologlcally } \\
\text { normal fetus at or beyond the } 10^{\circ} \text { week of gestation }\end{array}$ \\
\hline $\bar{x}$ & $\begin{array}{l}\text { D. One of more pre-term oirths of a morphologically normal } \\
\text { neonate }\end{array}$ \\
\hline$x$ & $\begin{array}{l}\text { C. Detore the 34" week of gestation becasuse of: D) eclampsia or } \\
\text { severe pre-eclampsia or (i) recognized features of placental } \\
\text { insufficiency }\end{array}$ \\
\hline \multirow[t]{2}{*}{$x$} & $\begin{array}{l}\text { d. Three of more unexplained consecutve spontaneous } \\
\text { miscarriages before the } 10^{\circ} \text { week of gestation, with maternal } \\
\text { anatomic or hormonal abnormalifes and paternal and maternal } \\
\text { chromosomal causes excluded }\end{array}$ \\
\hline & Laboratory criteria \\
\hline$\checkmark$ & $\begin{array}{l}\text { 1. Lupus anticosgutant (LR) present in plasma, on two or more } \\
\text { occasions at least } 12 \text { weeks apart }\end{array}$ \\
\hline $\bar{x}$ & 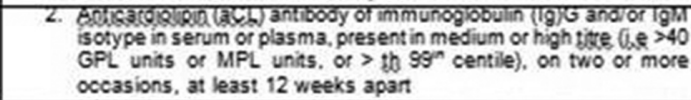 \\
\hline $\bar{x}$ & 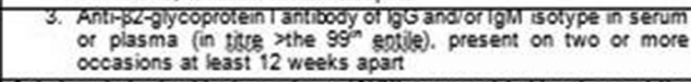 \\
\hline $\bar{x}$ & $\begin{array}{l}\text { Antiphos pholipo antroody syndome (ARPS) is presentr at least one of the } \\
\text { clinical criteria and one of the laboratory criteria are met }\end{array}$ \\
\hline
\end{tabular}

for an autoimmune disease such as APAS to prevent maternal and fetal complications is recommended.

Presenting a 29 year old, primigravid of 32 weeks age of gestation was admitted due to epigastric pain, hypotension and decreased fetal movement. She delivered to a dead male neonate via Emergency Low Segment Transverse Caesarean Section. Hemoperitonium and active bleeding from the liver were noted intraoperatively.

Methods Patient was managed as spontaneous hepatic rupture. APAS was considered and diagnosed via the Revised SAPPORO Criteria. HELLP syndrome and pre-eclampsia were ruled out by clinical and laboratory parameters. 